\title{
Exploration of Modulated Genetic Circuits Governing Virulence Determinants in Staphylococcus aureus
}

\author{
Rekha Arya $^{1} \cdot$ S. Adline Princy ${ }^{1}$
}

Received: 6 July 2015/ Accepted: 15 October 2015/Published online: 22 October 2015

(C) Association of Microbiologists of India 2015

\begin{abstract}
The expression of virulence genes in the human pathogen Staphylococcus aureus is strongly influenced by the multiple global regulators. The signal transduction cascade of these global regulators is accountable for recognizing and integrating the environmental cues to regulate the virulence regulon. While the production of virulent factors by individual global regulators are comparatively straightforward to define, auto-regulation of these global regulators and their impact on other regulators is more complex process. There are several reports on the production of virulent factors that are precisely regulated by switching processes of multiple global regulators including some prominent accessory regulators such as agr, sae and sar which allows $S$. aureus to coordinate the gene expression, and thus, provide organism an ability to act collectively. This review implicates the mechanisms involved in the global regulation of various virulence factors along with a comprehensive discussion on the differences between these signal transduction systems, their auto-induction and, coordination of classical and some comparatively new bacterial signal transduction systems.
\end{abstract}

Keywords Staphylococcus aureus - Global regulators · Virulence determinants - Signal transduction system

S. Adline Princy

adlineprinzy@biotech.sastra.edu

1 Quorum Sensing Laboratory, Centre for Research on Infectious Diseases, School of Chemical and Biotechnology, SASTRA University,

Thirumalaisamudram, Thanjavur 613 401, Tamil Nadu, India

\section{Introduction}

\section{Staphylococcus aureus Pathogenesis}

Multi-drug resistant (MDR) S. aureus is associated with higher morbidity and mortality in both nosocomial as well as community settings [1]. Infections caused by MDR S. aureus especially in hospital settings is evidently challenging to treat due to the development of resistance to multiple antimicrobial agents as a result of both intrinsic and acquired mechanisms [2, 3]. Furthermore, the emergence of vancomycin resistance among $S$. aureus absence of new antibacterials have made treatment of these isolates very difficult. Hence, the knowledge about the regulation of virulence gene expression and host pathogen interaction can open new avenues towards the development of novel antivirulent agents to conquer the problem of drug resistance among bacteria. The interaction between $S$. aureus and the host in the course of infection is a dynamic battleground where the ingenious approaches of $S$. aureus for existence and localization run into head on with the challenging defenses of the host immune system. S. aureus expresses an extensive range of virulence factors that act in a synchronized manner and increases its capability to inhabit and cause various diseases in the hosts [4-6]. These virulence factors facilitate the biofilm formation, invasion, and colonization to overcome the host defense mechanisms [7]. Approximately all the $S$. aureus strains produce a set of toxins and enzymes like $\alpha, \beta, \gamma$ and $\delta$-hemolysins, proteases, nucleases and lipases to transform the host tissues into nutrients vital for their cell growth [8]. Staphylococcal pathogenicity ensue in a phase dependent fashion involving multiple virulence factors, each phase linking one or numerous explicit virulence factors with the exception of toxin-interceded infections for instance the toxic shock syndrome (TSS) [9]. 
Key developments using genetic approach have recognized that the global regulators often coordinately regulate the expression of these divergent virulence determinants. The virulence factors of $S$. aureus can be broadly categorized into exoproteins which majorly includes hemolysins and extracellular proteases and cell wall associated proteins such as adhesin. Numerous studies have been undertaken in the past to demonstrate that the $S$. aureus has developed a highly advance signal transduction systems which regulates the synchronized expression of multiple virulence genes. Earlier studies have revealed that the multiple drug resistant $S$. aureus is well equipped to resist antibiotics and also several regulatory loci is a key determinant to establish pathogenicity [10]. Understanding the sequential auto-induction of the genetic circuits regulating the virulence gene expression can provide critical information for specific inhibition of the loci that are accountable for the $S$. aureus pathogenicity and could possibly evolve an exciting therapeutic prospects. Several loci including agr, sar, sae and rot are important constituents for the expression of various toxins and the cell wall associated proteins in response to various stimuli to help the $S$. aureus to infect and inhabit host tissues. Hence, enhanced knowledge of roles and mechanism of actions of every locus is significant for the successful prognosis of individuals suffering from the staphylococcal infections. This review highlights the role and molecular basis of well-characterized loci in $S$. aureus responsible for the virulence gene expression (Fig. 1; Table 1). A comprehensive study of each locus could open a way to identify inhibitors as antivirulent agents rather antimicrobials that could negatively regulate the expression of various virulence genes and may be supportive for the expansion of unique therapies for MDR S. aureus caused infections [11].

\section{agr: The Global Regulator}

The accessory gene regulator (agr) locus is extensively studied in $S$. aureus and controlled by two distinct promoters P2 and P3 which encodes a two-component histidine kinase signal-transduction system comprising of two different transcription elements [12]. The hld RNA transcript act as a regulatory molecule of the agr system is encoded by the P3 promoter, while the divergent four ORF labeled as $\operatorname{agr} B, \operatorname{agrD}, \operatorname{agrC}$, and $\operatorname{agrA}$ is driven by the $\mathrm{P} 2$ promoter [13]. The signal transduction is mediated by an auto-inducing peptide produced and matured by $a g r B$ and $\operatorname{agrD}$ that activates AgrC which is a two component histidine kinase receptor $[14,15]$. The key virulence regulator, agr prompts distinct alterations in the gene expression which is the cell density dependent mechanism termed as quorum sensing [16, 17]. The accessory gene regulator

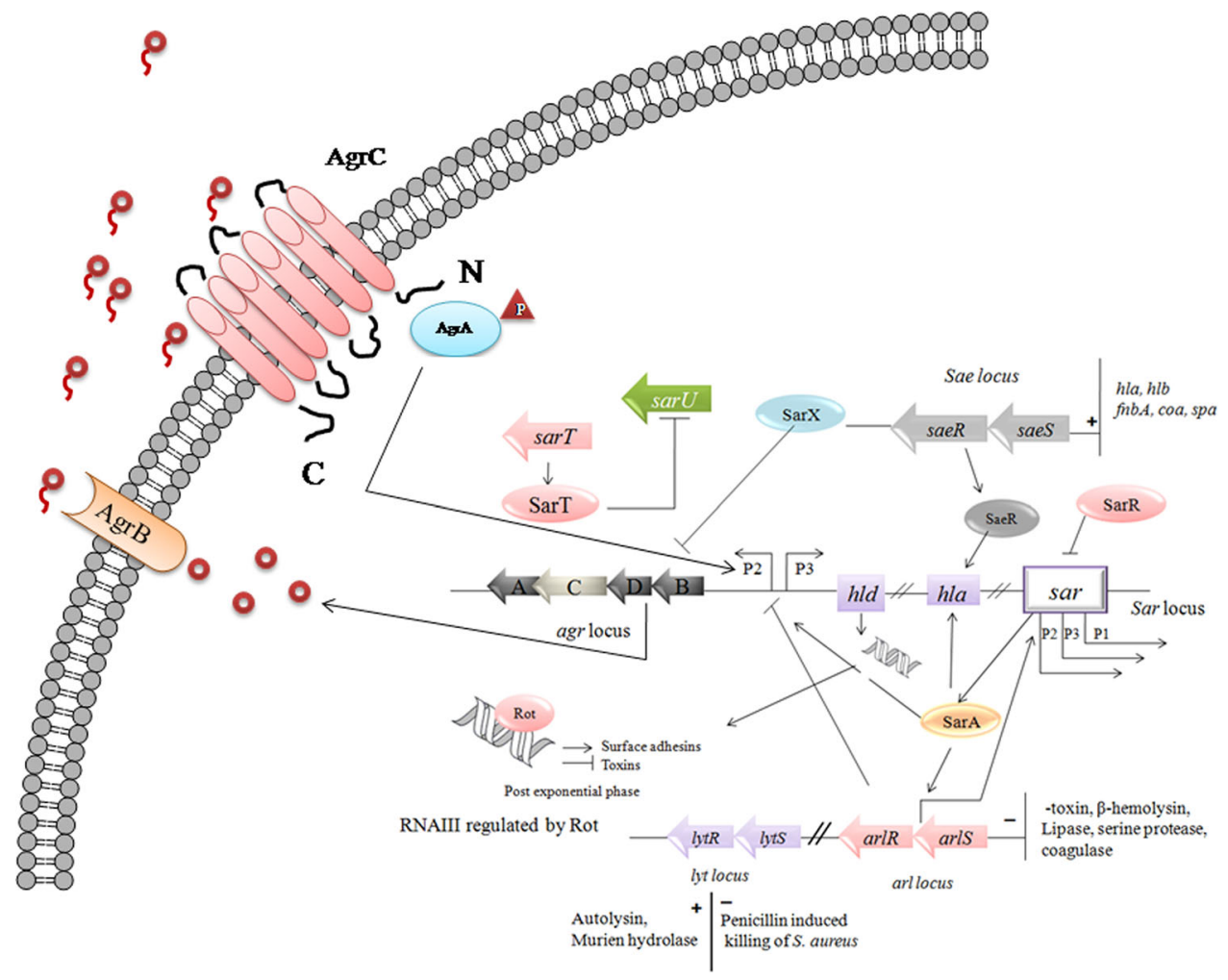

Fig. 1 The intricate molecular mechanism for the virulence gene expressions in Staphylococcus aureus. Positive and negative symbols depict the up-regulation and down-regulation of virulence determinants 
Table 1 The pathogenic islands regulate virulence determinants of $S$. aureus

\begin{tabular}{lllllllll}
\hline Virulence determinants & \multicolumn{4}{l}{ Pathogenic Islands } \\
\cline { 2 - 5 } & agr & sar & sae & arl & rot & KdpDE & lyt
\end{tabular}

Enterotoxins

Enterotoxin B

Enterotoxin C

Enterotoxin D

Cytotoxins

$\alpha$-Hemolysin

$\beta$-Hemolysin

$\delta$-Hemolysin

$\Upsilon$-Hemolysin

Exfoliatins

Exfoliatin A

Exfoliatin B

Toxic shock toxin-1

Exotoxins

Set8

Set9

Enzymes

Fatty acid modifying enzyme

FemA

FemB

Aureolysin (metalloprotease)

Hyaluronic lyase

Lipase

Phospholipase

Serine protease

Staphylokinase

V8 protease

Panton-valentine leukocidin

Coagulase

Nuclease

DNase

Autolysins

Murein hydrolase

Peptidoglycan hydrolase

Surface proteins

Collagen binding proteins

Clumping factor B

Fibronectin binding protein A

Fibronectin binding protein $\mathrm{B}$

Protein A

Capsular polysaccharides

Type 5

Type 8

$\begin{array}{ll}+ & + \\ + & \\ +\end{array}$

$+$

$+$

$+$

$+$

$+$

$+$

$+$

$-$

$+\quad+$

$+$

$+$

$\pm$

$+$

$+\quad+$

$+\quad+$

$+$

$+$

$+\quad+$

$+$

$+$

$+$

$+$

$+$

$+$

$+$

+
+

$+$

$+$

$+$

$$
+
$$

$+$

.

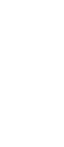


$(a g r)$ is known to modulate an extensive array of virulence factors such as nucleases, proteases, lipases and expression of surface binding proteins [18, 19]. The adaptation to fine tuning of the gene expressions is thought to determine the production of explicit arrays of virulence factors at various phases to establish infection. When the bacterial population is low, the expression of adherence proteins are triggered for the attachment to host tissue and toxins are produced once the infection is established [20-22]. Sensible initiation of $a g r$ in vivo and its prominence for pathogenicity of $S$. aureus has been validated. Decrease in the secretion of extracellular toxins and an increased production of fibronectin binding protein $(f n b)$, protein A and other cell-surface associated proteins were observed in agr null strains $[23,24]$. The expression of agr locus also provides a simultaneous impact on colony spreading and virulent determinants and are regulated by the environmental cues [25]. The colony spreading in $S$. aureus is negatively regulated by the $\delta$-hemolysin and the experimental data also demonstrated that hld mutant strains confirm the prominent colony spreading than the parental level [26]. An aminoswapping in vitro analysis revealed that virulence gene expression is suppressed by the phenylalanine whereas the hemolytic and protease activity are being repressed by the aspartic acid [27].

\section{sarA: Pleiotropic Regulator}

The synthesis of polysaccharide intercellular adhesion (PIA) and the subsequent formation of the S. aureus biofilm is governed by a pleiotropic regulator known as SarA. The sar operon comprises of three overlapping regions specifically $\operatorname{sar} A, \operatorname{sar} C$ and $\operatorname{sar} B$ with a size of $0.58,0.85$ and $1.15 \mathrm{~kb}$ respectively. These transcripts encode a $14.5 \mathrm{kDa}$ SarA protein and governed by three diverse promoters namely $\mathrm{P} 1, \mathrm{P} 3$ and $\mathrm{P} 2$ respectively [28, 29]. It is evident from the crystal structure of SarA that binds as homodimers to the AT rich conserved region of the regulatory domain (promoter) in the DNA. The SarA is a DNA binding protein where each monomer is a 124 residues polypeptide chain that comprises of four $\alpha$-helices, a C-terminal loop and a short $\beta$-hairpin [30]. SarA, a wellcharacterized regulator alter the expression of the various virulence genes and also shows effective interactions on the intergenic region of $\mathrm{P} 2$ and $\mathrm{P} 3$ promoter to activate the agr operon [31, 32]. In addition to the $a g r$ intergenic promoter SarA can also directly interact to the conserved regions of the various other factors those are independent of $a g r$ locus including the cell wall associated proteins like fibronectin-binding protein, adhesins, protein-A and exoproteins are known as sar boxes. It was observed that sarA is expressed in all the growth phases of the $S$. aureus and the expression is modulated by various other factors [33].
A pleotropic effect was observed on the expression of the cell wall associated and several other virulence proteins when a transposon (Tn91-LTV1) inserted into the sarA region which was distinct from the agr locus in the $S$. aureus chromosome. It also modulates the synthesis of several other virulence factors including extracellular proteases [34]. The study on 105 strains of $S$. aureus has revealed that the agr mutant strains increased the biofilm formation and failed to synthesize $\delta$-toxin than sarA mutated strains $[35,36]$. The study also demonstrated the limitation of those strains to form form biofilm due to the synthesis of $\delta$-toxin to act as a surfactant. Significantly, it is also evident that the hld gene for the $\delta$-toxins encoded within the agr dependent RNAIII regulatory molecule. There are limited data exist to explore the anti-biofilm potential of various SarA based inhibitors [37, 38].

\section{sae: S. aureus Accessory Element}

The sae operon involves four distinct ORFs known as sae $P Q R S$ where a response regulator and a sensor histidine kinase are encoded by saeR and saeS respectively and together they forms a two component regulatory system [39]. The operon functions are significantly regulated by another two open reading frames, saeP and sae $Q$ which are located upstream to saeRS but the activation of these two component regulatory system is not extensively studied. Similar to the other two component signal transduction system, SaeS act as a trans membrane histidine kinase sensor protein which auto-phosphorylates in the presence of environmental signals and consecutively triggers $s a e R$, a response regulator. The triggered saeR identify the promoter sequences of target genes and hence, may work as a transcriptional regulator. Sae is a vital global regulator and studies have shown that the deletion of $3.4 \mathrm{~kb}$ sae regions resulted in the decreased expression of many virulence factors including bacterial adhesion and serine proteases. It is also established from various studies that the mutation in sae results for the synthesis of extreme suppression of coagulase and to some extent decreased levels of protein A [40, 41]. Varied environmental cues, $\mathrm{pH}$, salinity and other global regulators of $S$. aureus such as agr has an impact on the pattern of the sae expression [42, 43]. It was observed that the sub-inhibitory concentrations of antibiotics such as glycopeptides and $\beta$-lactams activate the expression of sae while antibiotics such as clindamycin impede the expression. Hence, sae seems to be an essential modulator that regulates the expression of key virulence factors for instance hla, coding for coagulase (coa) and fnbA. In vivo neonatal mouse model and mutational analysis strongly suggested that saeRS is a major regulator for the exfoliation activity [44]. However, the influence of other global regulators on sae is partially conflicting, which may be 
possibly due to differences in the strains used in the various studies.

\section{lyt Regulon: The Two Component Histidine Kinase System}

To understand the LytSR function, the S. aureus chromosome was cloned and the structural and functional similiarity was compared with the two component histidine kinase receptor, lytR and lytS [45]. The sequencing also revealed the presence of two additional ORFs downstream from $\operatorname{lyt} R$, termed as $\operatorname{lrg} A$ and $\operatorname{lrg} B$ but the functionality is still unclear [46]. But, it was observed that during the stationary growth phase of $S$. aureus, the LytRS governs $\operatorname{lrg} A B$ operon and explicitly intricate in negative regulation of the activity of murein hydrolase and lysis induced by penicillin. The data from mutation studies have revealed that the mutation in the lytS gene results in an increased production of autolysis.

A difference in the murein hydrolase activity was detected in the lyt $S$ mutant as apparent in the parental strain and hence, the integrity of the cell wall is linked with the lytRS regulon to control the activity via $\operatorname{lrg} A B$ [47]. The amino acid sequencing revealed that the $\operatorname{lrg} \mathrm{A}$ is a $16.3 \mathrm{kDa}$ protein that contain 148 residues whereas the additional ORF, $\operatorname{lrg} B$ that encodes a $25.1 \mathrm{kDa}$ protein $(\mathrm{LrgB}) \mathrm{com}$ prised of 233 residues [48]. Earlier reports demonstrated that lytSR locus play an imperative role during biofilm formation by controlling cell lysis and mediated by the $\operatorname{lrg} A B$ and eDNA [49].

\section{arlRS: The Autolysis-Related Locus}

The two component regulatory system, arlRS is responsible for the virulence gene expression including adhesion and autolysis [50]. The ArlRS system has identified as a first regulator to influence the biofilm formation, extracellular proteolytic activity, autolysis and the ArlR, also positively modulate the agr system [51]. Mutation analysis has revealed that the $\operatorname{arl}$-arlS two components syste are also responsible for the production of several secreted proteins in $S$. aureus. The arl locus is transcribed during the exponential growth phase of $S$. aureus and it consists of two overlapping ORFs and transcribed together into a $2.7 \mathrm{~kb}$ mRNA. The locus comprises a $52.4 \mathrm{kDa}$ sensor protein, ArlS and a $25.5 \mathrm{kDa}$ ArlR regulator protein have a higher sequence similarity with PhoB-OmpR family proteins. Studies have shown that the mutation in $\operatorname{arl} S$, a DNA binding protein increases the activity of peptidoglycan hydrolase and leads to its autolysis. It encompasses a conserved C-terminal domain that binds to the upstream of the target gene promoters and most likely regulates the cell adherence and division. The arl regulon is multifaceted regulatory system to either directly or circuitously via $a g r$ and sar operon which modulate the expression of various virulence genes including the serine proteases, protein $\mathrm{A}$, coagulase etc. These results indicate that the inactivation of agr operon and hence, down-regulation of the genes activated by the RNAIII during cell adherence and growth is influenced by the Arl two component regulatory system. It was also observed that the Arl also regulate genes through $m g r A$-dependent pathway and up-regulates the production of capsule in the S. aureus at the transcriptional level [52]. The exfoliative toxins are positively regulated by the $\operatorname{arlRS}$ system and the ArlRS also down regulate the autolysis in the methicillin-sensitive $S$. aureus strains, but when the arlRS was inactivated it had no impact on autolysis of methicillin-resistant $S$. aureus [53].

\section{KdpDE: An Universal Communication Network}

Autoinducer 2 (AI-2) is a small diffusible molecule widely present in the gram positive and gram negative bacteria and it is also referred as the universal language for the interspecies communication [54] AI-2 is synthesized by the LuxS enzyme during the metabolic pathway. AI-2 is an interconverting compound and derived from the 4,5-dihydroxy-2,3-pentanedione (DPD) to further cyclizes and form two epimeric furanones $(2 \mathrm{R}, 4 \mathrm{~S})$ and $(2 \mathrm{~S}, 4 \mathrm{~S})$-2,4-dihydroxy-2-methyldihydrofuran-3-one (R-and S-DHMF). LuxS/AI-2 system is more evidently investigated in the Staphylococcus epidermidis where the luxS gene was functional to regulate the transcription of polysaccharide intercellular adhesion gene ica for the biofilm formation and it also modulates the virulence gene expression [55, 56].

AI-2 quorum sensing in S. aureus is associated with the two components KdpDE system to regulate the virulence determinants as well as capsular polysaccharide synthesis, an important cell wall constituent [57], during the invasive process it interacts with the host immune system and allows the microbes to resist from phagocytosis. Moreover, 11 types of capsular polysaccharides are reported, most of the $S$. aureus isolates belongs to CP5 or CP8. Earlier experiments revealed that cap operon transcription in the $S$. aureus NCTC 8325 is regulated by the array of regulatory loci they are sarA, agr, sae, arlRS, sbcDC, ccpA, mgr, yabJ-spoVG [58, 59]. Furthermore, it has also demonstrated that the diverse environmental factors also regulate the CP expression. Research data revealed that CP5 and CP8 used as a vaccine development and generated specific antibodies and showed protection against $S$. aureus infections. KdpDE system was first described in E. coli where the $\mathrm{KdpE}$ and $\mathrm{KdpD}$ proteins regulate the production of Kdp-ATPase, it is a high affinity $\mathrm{K}^{+}$Transporter [60]. 
KdpDE comprise of two proteins, a response regulator and its sensor histidine kinase. The histidine kinase sensor perceives the environmental signals and initiates the phosphorylation mechanism. The recent data demonstrated that the inactivation of KdpDE showed the variation in the virulence gene transcription comprises spa, cap, hla, aur, $g e h$, and $h \lg B$ and electromobility shift assay (EMSA) also revealed that $\mathrm{KdpE}$ directly binds to these genes promoter to modulate the transcription [61, 62]. Allelic replacement experiment demonstrated that the $S$. aureus luxS regulate the CP5 gene transcription via AI-2 quorum sensing pathway but the exact mechanism that how LuxS/AI-2 interact with $\mathrm{KdpDE}$ and activate the pathway is still require further studies. Accessory gene regulator (agr) also plays an important role to trigger the $k d p D E$ by RNAIII, transcriptional experiment revealed that $k d p D$ and $k d p E$ level were increased when the cell were grown to the post exponential growth phase as it suggests that the Agr quorum sensing influence the transcription of $k d p D E$. Furthermore electromobility shift assay also demonstrated that the rot protein specifically binds to the promoter of $k d p D$ (Fig. 2).

\section{Repressor of Protein (rot)}

The repressor of protein (rot) is a transcription repressor to negatively regulate the production of the virulence factors such $\alpha$-toxin and protease via the $a g r$ operon. It has a 498 bp open reading frame (ORF) that encodes a predicted 161 amino acids protein with a molecular mass of $15.6 \mathrm{kDa}$ [63] and has a partial sequence resemblance with AgrA and SarA. Rot is a global regulator of various genes and encode exotoxins for instance proteases and lipases to play a vital role in the invasion of bacterial cells in the host tissues. The experiment has revealed that the Rot modulates the expression of at least 146 genes where some genes such as spa, sspB and sspC are up-regulated while $h l a$ and $\gamma$-hemolysin gene is suppressed by Rot $[64,65]$. These results clearly indicate that the Rot has a suppressive effect on the expression of agr and it probably inhibits the expression of RNAIII, however the regulatory pathway intricate remains unrevealed. The Rot inhibits the expression of the target genes by impeding their transcription during the exponential phase of $S$. aureus growth by interacting with genes within the promoter region. It also proposed that Rot up-

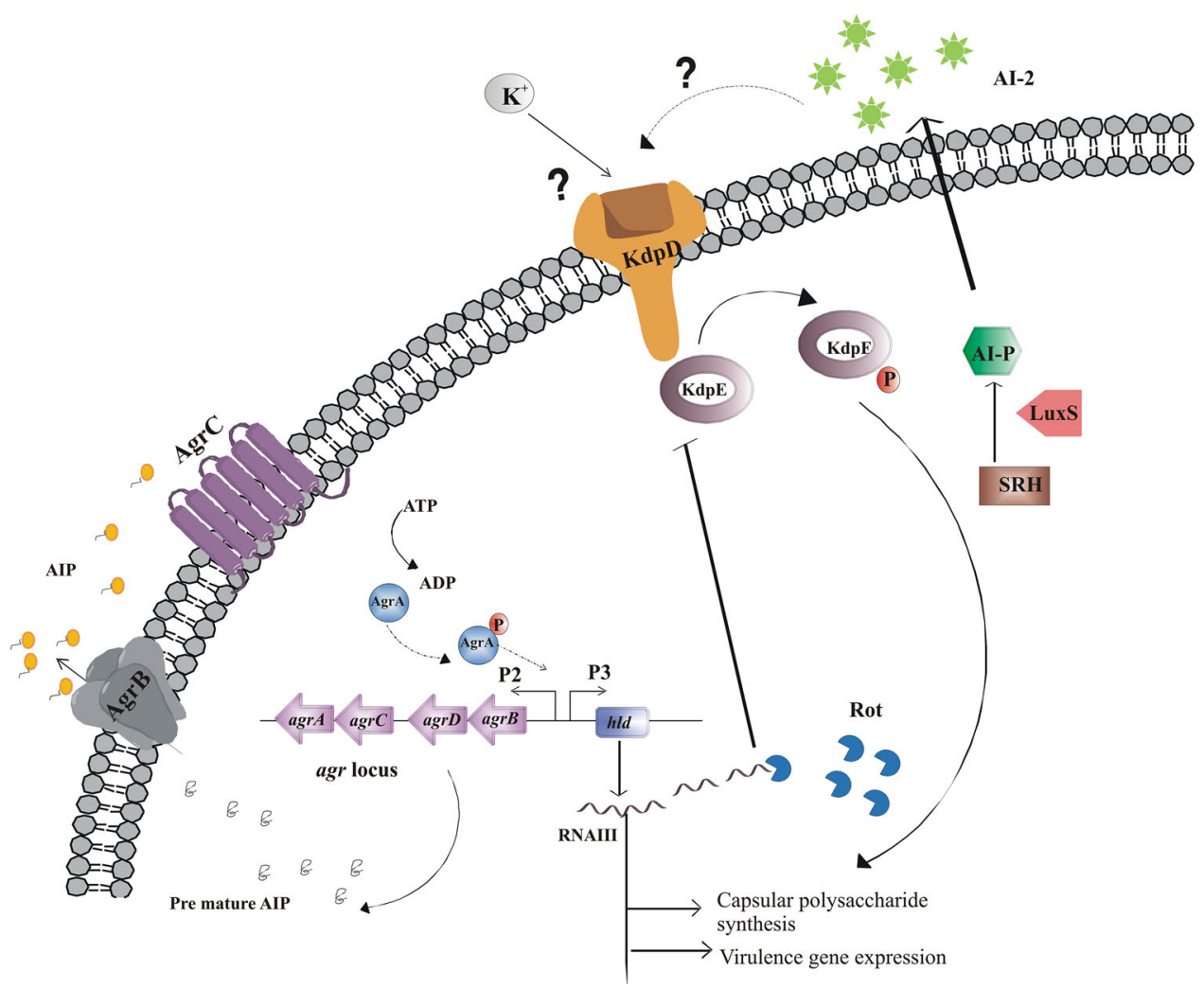

Fig. 2 The Agr quorum sensing circuit coordinates with the KdpDE two component networks to regulate the capsular polysaccharide production in $S$. aureus 
regulates the expression of adhesins and hence promotes the $S$. aureus cell proliferation. The study on the production of $\alpha$-toxin showed that re-establishment of the rot expression totally blocks the expression of this gene and disruption of rot in the $a g r$ mutant strains partially reinstates the activity. Since, the expression of various virulence genes in $S$. aureus is modulated by Rot, it is very essential to comprehend the regulatory pathway and elucidate the linking of Rot with other key virulence determinants.

\section{Conclusion}

Sustained occurrence of the MDR S. aureus in the community is a serious problem that deserves enhanced attention in the diagnosis and to identify novel therapeutic targets to curb staphylococcal infections. As discussed above, the $S$. aureus has developed advanced signaling systems that plays an imperative role in staphylococcal infections and pathogenesis. The virulence gene expression in $S$. aureus is subjected to constraints at various levels by these global regulatory architectures (two-component systems) that that sense several environmental cues to configure optimal rates of toxin production. The complexity in the fine tuning of the genetic circuits support the viability and pathogenicity of multi-drug resistant $S$. aureus in disease progression. The disease progression involves various virulence factors that provide a discrete benefit for the cell to cell proliferate and establish cell-density dependent inter-/or intra- species communication to mediate biofilm and pathogenesis [66]. Also, the bacterial cells have shown tremendous advances and evolution in response to their environment such as development of antibacterial resistant mechanism. So our understanding to those genetic circuits with overlapping responses to dynamically control the expression of various factors, regulons and stimulators will probably lead to develop new strategies to combat staphylococcal infections.

Acknowledgments The authors thank the University Grant Commission, Government of India for providing fellowship under Rajiv Gandhi National Fellowship Scheme, and appreciate the excellent management of SASTRA University.

\section{References}

1. McAdam PR, Templeton KE, Edwards GF, Holden MT, Feil EJ, Aanensen DM, Bargawi HJ, Spratt BG, Bentley SD, Parkhill J, Enright MC, Holmes A, Girvan EK, Godfrey PA, Feldgarden M, Kearns AM, Rambaut A, Robinson DA, Fitzgerald JR (2012) Molecular tracing of the emergence, adaptation, and transmission of hospital-associated methicillin-resistant Staphylococcus aureus. Proc Natl Acad Sci 109:9107-9112. doi:10.1073/pnas. 1202869109
2. Bishara J, Goldberg E, Leibovici L, Samra Z, Shaked H, Mansur N, Paul M (2012) Healthcare-associated vs. hospital-acquired Staphylococcus aureus bacteremia. Int J Infect Dis 6:e457-e463. doi:10.1016/j.ijid.2012.02.009

3. Kalia VC (2014) Microbes, antimicrobials and resistance: the battle goes on. Indian J Microbiol 54:1-2. doi:10.1007/s12088013-0443-7

4. Banchereau R, Jordan-Villegas A, Ardura M, Mejias A, Baldwin N, Xu H, Saye E, Rossello-Urgell J, Nguyen P, Blankenship D, Creech CB, Pascual V, Banchereau J, Chaussabel D, Ramilo O (2012) Host immune transcriptional profiles reflect the variability in clinical disease manifestations in patients with Staphylococcus aureus infections. PLoS ONE 7:e34390. doi:10.1371/journal. pone. 0034390

5. Bohach GA, Fast DJ, Nelson RD, Schlievert PM (1990) Staphylococcal and streptococcal pyrogenic toxins involved in toxic shock syndrome and related illnesses. Crit Rev Microbiol 17:251-272. doi:10.3109/10408419009105728

6. Dinges MM, Orwin PM, Schlievert PM (2000) Exotoxins of Staphylococcus aureus. Clin Microbiol Rev 13:16-34. doi:10. 1128/CMR.13.1.16-34.2000

7. Lowy FD (1998) Staphylococcus aureus infections. N Engl J Med 339:520-532. doi:10.1056/NEJM199808203390806

8. Huseby M, Shi K, Brown CK, Digre J, Mengistu F, Seo KS, Bohach GA, Schlievert PM, Ohlendorf DH, Earhart CA (2007) Structure and biological activities of beta toxin from Staphylococcus aureus. J Bacteriol 189:8719-8726. doi:10.1128/JB. 00741-07

9. Lin YC, Anderson MJ, Kohler PL, Strandberg KL, Olson ME, Horswill AR, Schlievert PM, Peterson ML (2011) Proinflammatory exoprotein characterization of toxic shock syndrome Staphylococcus aureus. Biochemistry 50:7157-7167. doi:10.1021/ bi200435n

10. Gordon RJ, Lowy FD (2008) Pathogenesis of methicillin-resistant Staphylococcus aureus infection. Clin Infect Dis 46:S350-S359. doi:10.1086/533591

11. Kalia VC, Purohit HJ (2011) Quenching the quorum sensing system: potential antibacterial drug targets. Crit Rev Microbiol 37:121-140. doi:10.3109/1040841X.2010.532479

12. Ji G, Beavis R, Novick RP (1997) Bacterial interference caused by autoinducing peptide variants. Science 276:2027-2030. doi:10.1126/science. 276.5321 .2027

13. Balban N, Novick RP (1995) Autocrine regulation of toxin synthesis by Staphylococcus aureus. Proc Natl Acad Sci USA 92:1619-1623. doi:10.1073/pnas.92.5.1619

14. George Cisar EA, Geisinger E, Muir TW, Novick RP (2009) Symmetric signalling within asymmetric dimers of the Staphylococcus aureus receptor histidine kinase AgrC. Mol Microbiol 74:44-57. doi:10.1111/j.1365-2958.2009.06849.x

15. Lina G, Jarraud S, Ji G, Greenland T, Pedraza A, Etienne J, Novick RP, Vandenesch F (1998) Transmembrane topology and histidine protein kinase activity of AgrC, the agr signal receptor in Staphylococcus aureus. Mol Microbiol 28:655-662. doi:10. 1046/j.1365-2958.1998.00830

16. Kalia VC (2015) Microbes: the most friendly beings? In: Kalia VC (ed) Quorum sensing vs quorum quenching: a battle with no end in sight, vol 1-5. Springer, India. doi:10.1007/978-81-3221982-8_1

17. Kumar P, Koul S, Patel SK, Lee JK, Kalia VC (2015) Heterologous expression of quorum sensing inhibitory genes in diverse organisms. In: Kalia VC (ed) Quorum sensing vs quorum quenching: a battle with no end in sight, pp 343-356. Springer, India. doi:10.1007/978-81-322-1982-8_28

18. Kalia VC, Raju SC, Purohit HJ (2011) Genomic analysis reveals versatile organisms for quorum quenching enzymes: acyl- 
homoserine lactone-acylase and-lactonase. Open Microbiol J 5:1-13. doi:10.2174/1874285801105010001

19. Kalia VC, Kumar P (2015) Potential applications of quorum sensing inhibitors in diverse fields. In: Kalia VC (ed) Quorum sensing vs quorum quenching: a battle with no end in sight. Springer, pp 359-370. doi:10.1007/978-81-322-1982-8_29

20. Nusrat H, Kushwah J, Joshi J, Raju SC, Kalia VC (2011) Diversity and polymorphism in AHL-lactonase gene (aiiA) of Bacillus. J Microbiol Biotechnol 21:1001-1011. doi:10.4014/ jmb.1105.05056

21. Kalia VC (2014) In search of versatile organisms for quorumsensing inhibitors: acyl homoserine lactones (AHL)-acylase and AHL-lactonase. FEMS Microbiol Lett 359:143. doi:10.1111/ 1574-6968.12585

22. Kalia VC, Kumar P (2015) The battle: quorum-sensing inhibitors versus evolution of bacterial resistance. In: Kalia VC (ed) Quorum sensing vs quorum quenching: a battle with no end in sight. Springer, pp 385-391. doi:10.1007/978-81-322-1982-8_31

23. Abdelnour A, Arvidson S, Bremell T, Ryden C, Tarkowski A (1993) The accessory regulator (agr) controls Staphylococcus aureus virulence in a murine arthritis model. Infect Immun 61:3879-3885

24. Ji G, Beavis RC, Novick RP (1995) Cell density control of staphylococcal virulence mediated by an octapeptide pheromone. Proc Natl Acad Sci USA 92:12055-12059

25. Tsompanidou E, Sibbald MJ, Chlebowicz MA, Dreisbach A, Back JW, van Dijl JM, Buist G, Denham EL (2011) Requirement of the agr locus for colony spreading of Staphylococcus aureus. J Bacteriol 193:1267-1272. doi:10.1128/JB.01276-10

26. Omae Y, Sekimizu K, Kaito C (2012) Inhibition of colonyspreading activity of Staphylococcus aureus by secretion of $\delta$ hemolysin. J Biol Chem 287:15570-15579. doi:10.1074/jbc. M112.357848

27. Arya R, Kannan RV, Shrivastava S, Princy SA (2011) Studies on the effect of amino acids to curb the pathogenesis of multidrug resistant Staphylococcus aureus. Asian J Chem 23:4295-4298

28. Bayer MG, Heinrichs JH, Cheung AL (1996) The molecular architecture of the sar locus in Staphylococcus aureus. J Bacteriol 178:4563-4570

29. Manna A, Cheung AL (2001) Characterization of sarR, a modulator of sar expression in Staphylococcus aureus. Infect Immun 69:885-896. doi:10.1128/IAI.69.2.885-896.2001

30. Schumacher MA, Hurlburt BK, Brennan RG (2001) Crystal structure of SarA, a pleiotropic regulator of virulence genes in $S$. aureus. Nature 409:215-219. doi:10.1038/35051623

31. Morfeldt E, Tegmark K, Arvidson S (1996) Transcriptional control of the agr-dependent virulence gene regulator, RNAIII, in Staphylococcus aureus. Mol Microbiol 21:1227-1237. doi:10. 1046/j.1365-2958.1996.751447.x

32. Arya R, Princy SA (2013) An insight into pleiotropic regulators Agr and Sar: molecular probes paving the new way for antivirulent therapy. Future Microbiol 10:1339-1353. doi:10.2217/fmb. 13.92

33. Manna AC, Bayer MG, Cheung AL (1998) Transcriptional analysis of different promoters in the sar locus in Staphylococcus aureus. J Bacteriol 180:3828-3836

34. Ballal A, Manna AC (2009) Expression of the sarA family of genes in different strains of Staphylococcus aureus. Microbiology 155:2342-2352. doi:10.1099/mic.0.027417-0

35. Arvidson S, Tegmark K (2001) Regulation of virulence determinants in Staphylococcus aureus. Int J Med Microbiol 291:159-170. doi:10.1016/j.femsre.2003.09.003

36. Vuong C, Saenz HL, Gotz F, Otto M (2000) Impact of agr quorum sensing system on adherence to polystyrene in Staphylococcus aureus. J Infect Dis 182:1688-1693. doi:10.1086/ 317606
37. Arya R, Princy SA (2012) Computational approach to design small molecule inhibitors and identify SarA as a potential therapeutic candidate. Med Chem Res 22:1856-1865. doi:10.1007/ s00044-012-0185-9

38. Arya R, Ravikumar R, Santhosh RS, Princy SA (2015) SarA based novel therapeutic candidate against Staphylococcus aureus associated with vascular graft infections. Front Microbiol 6:416. doi:10.3389/fmicb.2015.00416

39. Giraudo AT, Calzolari A, Cataldi AA, Bogni C, Nagel R (1999) The sae locus of Staphylococcus aureus encodes a two-component regulatory system. FEMS Microbiol Lett 177:15-22. doi:10. 1016/S0378-1097(99)00282-7

40. Giraudo AT, Mansilla C, Chan A, Raspanti C, Nagel R (2003) Studies on the expression of regulatory locus saein Staphylococcus aureus. Curr Microbiol 46:246-250. doi:10.1007/s00284002-3853-z

41. Goerke C, Fluckiger U, Steinhuber A, Bisanzio V, Ulrich M, Bischoff M, Patti JM, Wolz C (2005) The role of Staphylococcus aureus global regulators sae and $\operatorname{sig} B$ in virulence gene expression during device-related infection. Infect Immun 73:3415-3421. doi:10.1128/IAI.73.6.3415-3421.2005

42. Montgomery CP, Boyle Vavra S, Daum RS (2010) Importance of the global regulators Agr and SaeRS in the pathogenesis of CAMRSA USA300 infection. PLoS ONE 5:e15177. doi:10.1371/ journal.pone.0015177

43. Novick RP, Jiang D (2003) The staphylococcal sae RS system coordinates environmental signals with $a g r$ quorum sensing. Microbiology 149:2709-2717. doi:10.1099/mic.0.26575-0

44. Kato F, Kadomoto N, Iwamoto Y, Bunai K, Komatsuzawa H, Sugai M (2011) Regulatory mechanism for exfoliative toxin production in Staphylococcus aureus. Infect Immun 79:1660-1670. doi:10.1128/IAI.00872-10

45. Fournier B, Hooper DC (2000) A new two-component regulatory system involved in adhesion, autolysis and extracellular proteolytic activity of Staphylococcus aureus. J Bacteriol 182:3955-3964. doi:10.1128/JB.182.14.3955-3964.2000

46. Groicher KH, Firek BA, Fujimoto DF, Bayles KW (2000) The $S$. aureus $\operatorname{lrg} A B$ operon modulates murein hydrolase activity and penicillin tolerance. J Bacteriol 182:1794-1801. doi:10.1128/JB. 182.7.1794-1801.2000

47. Brunskill EW, Bayles KW (1996) Identification and molecular characterization of a putative regulatory locus that affects autolysis in Staphylococcus aureus. J Bacteriol 178:611-618

48. Brunskill EW, Bayles KW (1996) Identification of LytSR-regulated genes from Staphylococcus aureus. J Bacteriol 178:5810-5812

49. Zhu T, Lou Q, Wu Y, Hu J, Yu F, Qu D (2010) Impact of the Staphylococcus epidermidis LytSR two-component regulatory system on murein hydrolase activity, pyruvate utilization and global transcriptional profile. BMC Microbiol 10:287. doi:10. 1186/1471-2180-10-287

50. Fournier B, Klier A, Rapoport G (2001) The two component system ArlS-ArlR is a regulator of virulence gene expression in Staphylococcus aureus. Mol Microbiol 41:247-261. doi:10.1046/ j.1365-2958.2001.02515.x

51. Liang X, Zheng L, Landwehr C, Lunsford D, Holmes D, Ji Y (2005) Global regulation of gene expression by ArlRS, a twocomponent signal transduction regulatory system of Staphylococcus aureus. J Bacteriol 187:5486-5492. doi:10.1128/JB.187. 15.5486-5492.2005

52. Luong TT, Lee CY (2006) The arl locus positively regulates Staphylococcus aureus type 5 capsule via an mgrA-dependent pathway. Microbiology 152:3123-3131. doi:10.1099/mic.0.29177-0

53. Memmi G, Nair DR, Cheung A (2012) Role of ArlRS in autolysis in methicillin-sensitive and methicillin-resistant Staphylococcus 
aureus strains. J Bacteriol 194:759-767. doi:10.1128/JB.0626111

54. Waters CM, Bassler BL (2005) Quorum sensing: cell-to-cell communication in bacteria. Annu Rev Cell Dev Biol 21:319-346. doi:10.1146/annurev.cellbio.21.012704.131001

55. Kalia VC, Wood TK, Kumar P (2013) Evolution of resistance to quorum-sensing inhibitors. Microb Ecol 68:13-23. doi:10.1007/ s00248-013-0316-y

56. Kalia VC (2013) Quorum sensing inhibitors: an overview. Biotechnol Adv 31:224-245. doi:10.1016/j.biotechadv.2012.10. 004

57. Cluzel ME, Zanella Cléon I, Cozzone AJ, Futterer K, Duclos B, Molle V (2010) The Staphylococcus aureus autoinducer-2 synthase LuxS is regulated by Ser/Thr phosphorylation. J Bacteriol 192:6295-6301. doi:10.1128/JB.00853-10

58. Luong T, Sau S, Gomez M, Lee JC, Lee CY (2002) Regulation of Staphylococcus aureus capsular polysaccharide expression by agr and sarA. Infect Immun 70:444-450. doi:10.1128/IAI.70.2.444450.2002

59. Zhao L, Xue T, Shang F, Sun H, Sun B (2010) Staphylococcus aureus AI-2 quorum sensing associates with the KdpDE twocomponent system to regulate capsular polysaccharide synthesis and virulence. Infect Immun 78:3506-3515. doi:10.1128/IAI. 00131-10

60. Ballal A, Basu B, Apte SK (2007) The Kdp-ATPase system and its regulation. J Biosci 32:559-568
61. Kalia V, Kumar P, Pandian S, Sharma P (2015) Biofouling control by quorum quenching. In: Kim SK (ed) Handbook of marine biotechnology. Springer, Heidelberg, pp 431-440. doi:10. 1007/978-3-642-53971-8_15

62. Xue T, You Y, Hong D, Sun H, Sun B (2011) The Staphylococcus aureus KdpDE two-component system couples extracellular $\mathrm{K}+$ sensing and Agr signaling to infection programming. Infect Immun 79:2154-2167. doi:10.1128/IAI.01180-10

63. Mc Namara, Milligan Monroe PJ, Khalili KC, Proctor RA (2000) Identification, cloning, and initial characterization of rot, a locus encoding a regulator of virulence factor expression in Staphylococcus aureus. J Bacteriol 182:3197-3203. doi:10.1128/JB.182. 11.3197-3203.2000

64. SaidSalim B, Dunman PM, McAleese FM, Macapagal D, Murphy E, McNamara Arvidson PJ, Foster S, Projan TJ, Kreiswirth BN (2003) Global regulation of Staphylococcus aureus genes by Rot. J Bacteriol 185:610-619. doi:10.1128/JB.185.2.610-619.2003

65. Hsieh HY, Tseng CW, Stewart GC (2008) Regulation of Rot expression in Staphylococcus aureus. J Bacteriol 190:546-554. doi:10.1128/JB.00536-07

66. Schlievert PM, Strandberg KL, Lin YC, Peterson ML, Leung DY (2010) Secreted virulence factor comparison between methicillinresistant and methicillin-sensitive Staphylococcus aureus, and its relevance to atopic dermatitis. J Allergy Clin Immunol 125:39-49. doi:10.1016/j.jaci.2009.10.039 